\title{
Weight status and dietary fat modulate high density lipoprotein particle function in adolescents: results from a cross-sectional analysis and a randomised, placebo-controlled, crossover trial
}

\author{
A.M. McMorrow ${ }^{1}$, M.E. O’Reilly ${ }^{1}$, R.M. Connaughton ${ }^{1}$, E. Carolan ${ }^{2}$, D. O’Shea ${ }^{2}$, \\ F.E. Lithander ${ }^{3}$, F.C. McGillicuddy ${ }^{2}$ and H.M. Roche ${ }^{1}$ \\ ${ }^{1}$ UCD Conway Institute, University College Dublin, Ireland, ${ }^{2}$ Obesity Immunology Group, \\ Education and Research Centre, St Vincent's University Hospital, Dublin 4, Ireland and ${ }^{3}$ School of Public Health and \\ Nutrition, University of Canberra, Australia
}

This abstract was presented as the Cellular and Molecular Theme highlight.

Obese youth are at increased risk of developing cardiovascular disease in adulthood ${ }^{(1)}$. High density lipoprotein (HDL) particles facilitate efflux of cholesterol from lipid-laden arterial macrophages in atherosclerotic lesions, and deliver acquired lipid back to the liver for excretion ${ }^{(2)}$. This study aimed to characterise the impact of weight status and dietary fat on cholesterol efflux capacity in adolescents, which has not previously been described.

Cholesterol efflux from macrophages was determined as a metric of HDL function in a cross-sectional cohort of lean, overweight and obese adolescents $(n=82)$. Cholesterol efflux was assessed by incubating plasma HDL with ${ }^{3} \mathrm{H}$-cholesterol labelled $\mathrm{J} 774$ macrophages for $4 \mathrm{~h}$, from which ABCA1-dependent and ABCA1-independent efflux capacities were quantified. The effects of long chain (LC) n-3 polyunsaturated fatty acid (PUFA)-rich anti-inflammatory supplementation and a monounsaturated fatty acid (MUFA)-rich control supplement on efflux capacity were examined after an 8-wk randomised controlled crossover trial in overweight and obese adolescents $(n=52)$. Cholesteryl ester fatty acid composition was assessed as a biomarker of dietary fat intake.

Cross-sectional analysis demonstrated impaired total cholesterol efflux capacity in obese adolescents. Relative to lean adolescents, ABCA1-dependent efflux was impaired in overweight and obese adolescents with low cholesteryl ester n-3 PUFA status. However, HDL function was preserved in overweight and obese adolescents with high cholesteryl ester n-3 PUFA status, despite reduced HDL cholesterol concentration. Both LC n-3 PUFA-rich and MUFA-rich supplementation increased ABCA1-mediated cholesterol efflux in overweight and obese adolescents with impaired HDL function at baseline, independently of changes in HDL cholesterol or apolipoprotein A1 concentrations. Improved inflammatory profile, characterised by increased high molecular weight adiponectin and decreased complement component C3, was demonstrated in response to LC n-3 PUFA supplementation in adolescents with reduced baseline HDL function.

We demonstrate for the first time, impaired cholesterol efflux capacity in obese adolescents. However, both LC n-3 PUFA-rich anti-inflammatory supplementation and iso-energetic MUFA-rich supplementation partially rescued HDL function despite obesity. These novel findings illustrate the potential to attenuate cardiovascular disease risk in overweight and obese adolescents through dietary modification.

This research was supported by the National Children's Research Centre, Dublin, Ireland.

1. Rosenson RS, Brewer Jr HB, Davidson WS, et al. (2012) Circulation 125, 1905-1919.

2. Juonala M, Magnussen CG, Berenson GS, et al. (2011) NEJM 360, 1876-1885 